\title{
Quantitative proteomic, physiological and biochemical analysis of cotyledon, embryo, leaf and pod reveals the effects of high temperature and humidity stress on seed vigor formation in soybean
}

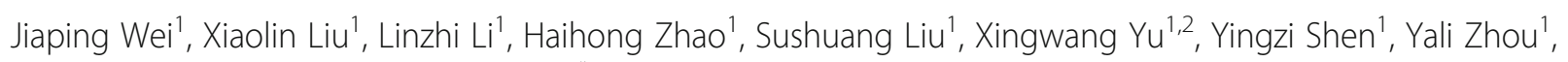
Yajing Zhu', Yingjie Shu ${ }^{1,3}$ and Hao $\mathrm{Ma}^{1 *}$

\begin{abstract}
Background: Soybean developing seed is susceptible to high temperature and humidity (HTH) stress in the field, resulting in vigor reduction. Actually, the HTH in the field during soybean seed growth and development would also stress the whole plant, especially on leaf and pod, which in turn affect seed growth and development as well as vigor formation through nutrient supply and protection.

Results: In the present study, using a pair of pre-harvest seed deterioration-sensitive and -resistant cultivars Ningzhen No. 1 and Xiangdou No. 3, the comprehensive effects of HTH stress on seed vigor formation during physiological maturity were investigated by analyzing cotyledon, embryo, leaf, and pod at the levels of protein, ultrastructure, and physiology and biochemistry. There were 247, 179, and 517 differentially abundant proteins (DAPs) identified in cotyledon, embryo, and leaf of cv. Xiangdou No. 3 under HTH stress, while 235, 366, and 479 DAPs were identified in cotyledon, embryo, and leaf of cv. Ningzhen No. 1. Moreover, 120, 144, and 438 DAPs between the two cultivars were identified in cotyledon, embryo, and leaf under HTH stress, respectively. Moreover, 120, 144, and 438 DAPs between the two cultivars were identified in cotyledon, embryo, and leaf under HTH stress, respectively. Most of the DAPs identified were found to be involved in major metabolic pathways and cellular processes, including signal transduction, tricarboxylic acid cycle, fatty acid metabolism, photosynthesis, protein processing, folding and assembly, protein biosynthesis or degradation, plant-pathogen interaction, starch and sucrose metabolism, and oxidative stress response. The HTH stress had less negative effects on metabolic pathways, cell ultrastructure, and physiology and biochemistry in the four organs of Xiangdou No. 3 than in those of Ningzhen No. 1, leading to produce higher vigor seeds in the former.

(Continued on next page)
\end{abstract}

\footnotetext{
* Correspondence: Lq-ncsi@njau.edu.cn

'State Key Laboratory of Crop Genetics and Germplasm Enhancement, Nanjing Agricultural University, Nanjing 210095, China

Full list of author information is available at the end of the article
}

(c) The Author(s). 2020 Open Access This article is licensed under a Creative Commons Attribution 4.0 International License, which permits use, sharing, adaptation, distribution and reproduction in any medium or format, as long as you give appropriate credit to the original author(s) and the source, provide a link to the Creative Commons licence, and indicate if changes were made. The images or other third party material in this article are included in the article's Creative Commons licence, unless indicated otherwise in a credit line to the material. If material is not included in the article's Creative Commons licence and your intended use is not permitted by statutory regulation or exceeds the permitted use, you will need to obtain permission directly from the copyright holder. To view a copy of this licence, visit http://creativecommons.org/licenses/by/4.0/. The Creative Commons Public Domain Dedication waiver (http://creativecommons.org/publicdomain/zero/1.0/) applies to the data made available in this article, unless otherwise stated in a credit line to the data. 
(Continued from previous page)

Conclusion: High seed vigor formation is enhanced by increasing protein biosynthesis and nutrient storage in cotyledon, stronger stability and viability in embryo, more powerful photosynthetic capacity and nutrient supply in leaf, and stronger protection in pod under HTH stress. These results provide comprehensive characteristics of leaf, pod and seed (cotyledon and embryo) under HTH stress, and some of them can be used as selection index in high seed vigor breeding program in soybean.

Keywords: Soybean, High temperature and humidity stress, Seed vigor, Proteomic, Ultrastructure, Physiology and biochemistry

\section{Background}

Temperature and humidity are two of the pivotal environmental factors associated with seed growth and development in field. High temperature and humidity condition can not only affect seed yield and quality, but also reduce seed vigor and storage capacity $[1,2]$, resulting in the preharvest seed deterioration. Seed vigor is a complex property that determines the seed's potential for rapid uniform germination and subsequent growth [3]. Moreover, HTH stress can also seriously interfere with the membrane compositions during seed development [4].

Soybean (Glycine max (L.) Merrill) is one of the most important legume crops and has a major impact on the global economies [5]. The vigor formation in developing soybean seeds generally starts from physiological maturity (R7) period. During this period, the developing seeds are susceptible to HTH stress, leading to the reduction of vigor. This situation occurs in many soybean production areas around the world [6-8]. Proteins are important structural components of cytoskeleton, membranes and cell wall in plants, which are responsible for most metabolic pathways and cellular processes in the seed. Hence, it would make senses to understand plant physiological processes by describing the proteome of a seed, a seed tissue, a specific cell type or a subcellular compartment [9].

So far, many researchers have investigated seed vigor at protein level [10-20]. Recently, several proteomic studies have focused on the effects of HTH stress on the vigor formation of developing soybean seed. For example, Wang et al. [21] analyzed the effects of $\mathrm{HTH}$ stress on the vigor formation of a pre-harvest seed deterioration-sensitive soybean cultivar by twodimensional electrophoresis (2-DE). Ma et al. [22] revealed the impacts of HTH stress on the vigor formation of a pre-harvest seed deterioration-resistant soybean cultivar by 2 -DE. Song et al. [23] reported a differentially proteomic analysis of developing seeds using a pair of pre-harvest seed deterioration-sensitive and -resistant soybean cultivars under HTH stress. In these studies, many proteins were found to be involved in the metabolic pathways and cellular processes that are potentially related to soybean seed vigor. Moreover, the pre-harvest deterioration-resistant cultivar possessed greater ability of ROS scavenging and cell defensing compared to the pre-harvest deterioration-sensitive cultivar under $\mathrm{HTH}$ stress. However, all these studies used the whole developing seeds as experimental materials to investigate the effects of HTH stress on soybean seed vigor formation. Actually, besides the developing seed, HTH condition would affect the whole plant, especially on leaf and pod. Soybean leaf is the primary site of photosynthesis, which contributes to the biosynthesis of plant biomass and energy [24, 25]. Soybean pod skin not only protects seeds, but also provides nutrients for seed growth and development through photosynthesis. Thus, it is imperative to consider the responses of leaf and pod when investigating seed vigor formation under $\mathrm{HTH}$ stress. In the present study, the comprehensive effects of $\mathrm{HTH}$ stress on seed vigor formation were evaluated using a pair of pre-harvest seed deterioration-sensitive and -resistant soybean cultivars at the levels of protein, ultrastructure, and physiology and biochemistry. The technique of isobaric tags for relative and absolute quantification (iTRAQ) was employed to detect the changes of proteins during stress. The aims are to find the major metabolic pathways and cellular processes involved in seed vigor formation in cotyledon, embryo, leaf and pod, and to lay a foundation for further revealing the mechanism of seed vigor formation under HTH stress.

\section{Results \\ Physiological responses to HTH stress}

No differences in net photosynthetic rates were found between the pre-harvest seed deterioration-resistant soybean cv. Xiangdou No. 3 and -sensitive cv. Ningzhen No. 1 under the normal condition (Fig. 1a). However, notably, the net photosynthetic rates of soybean cv. Xiangdou No. 3 were significantly $(p<0.01)$ higher than those of cv. Ningzhen No. 1 at the stress time points of 24 and 96 h (Fig. 1b).

\section{Microstructure comparison under HTH stress}

Transmission electron microscope (TEM) analysis was conducted using the cotyledons, embryos, leaves, and pods of two cultivars under the HTH stress (at $96 \mathrm{~h}$ ) and control (at $96 \mathrm{~h}$ ), respectively. Compared to the control 

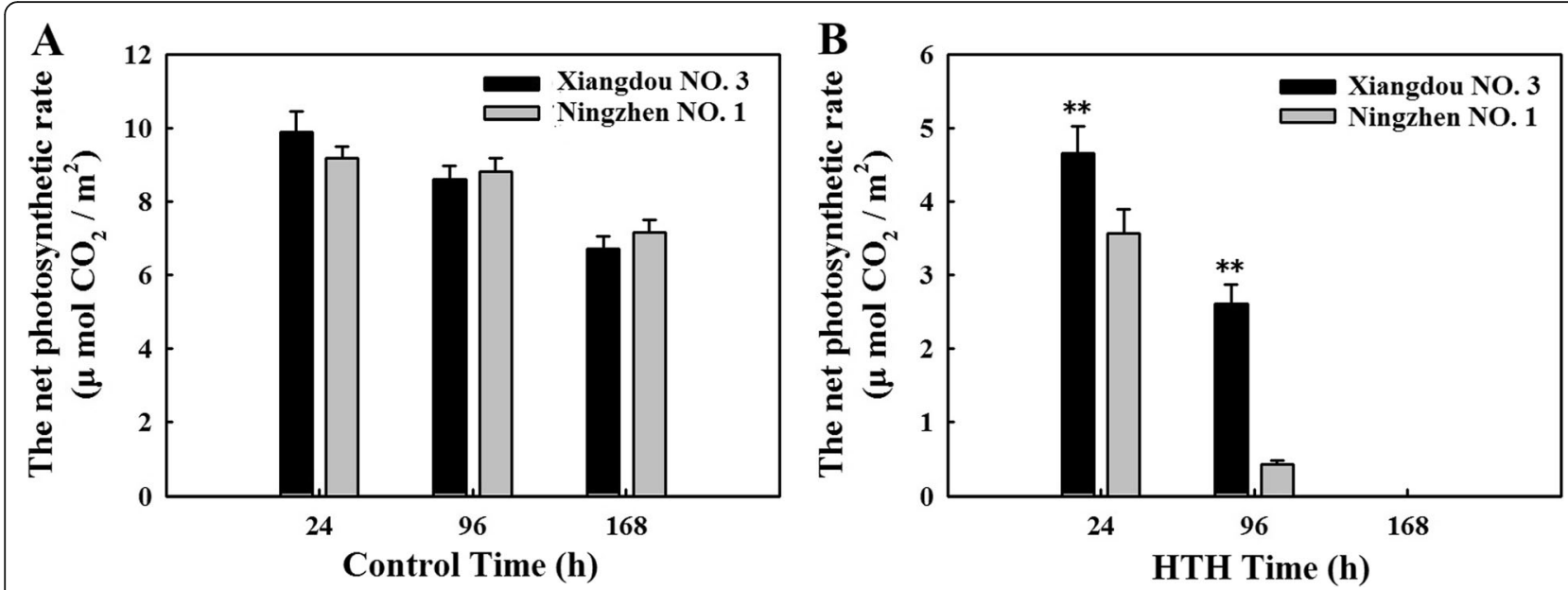

Fig. 1 The net photosynthetic rates of soybean cvs. Xiangdou No. 3 and Ningzhen No. 1. a, under the control condition; b, under the HTH stress. Values shown are means \pm SD from three biological replicates (**, $p<0.01$ )

(Fig. 2A), no obvious cyto-architecture changes were found in the cotyledon cell of cv. Xiangdou No. 3 (Fig. 2B). However, in the cotyledon cell of cv. Ningzhen No. 1, lipid bodies were found to be loosely arranged and a lot of cavities appeared under the HTH stress (Fig. 2C and D). Compared to the control (Fig. 2E), the number of protein bodies in the embryo cell of cv. Xiangdou No. 3 were accordingly reduced (Fig. 2F), while a larger number of protein bodies were disintegrated in the embryo cell of $\mathrm{cv}$. Ningzhen No. 1 under the HTH stress (Fig. 2G and H). The number of chloroplasts and starch grains in plant mesophyll cell were found to be decreased severely in both

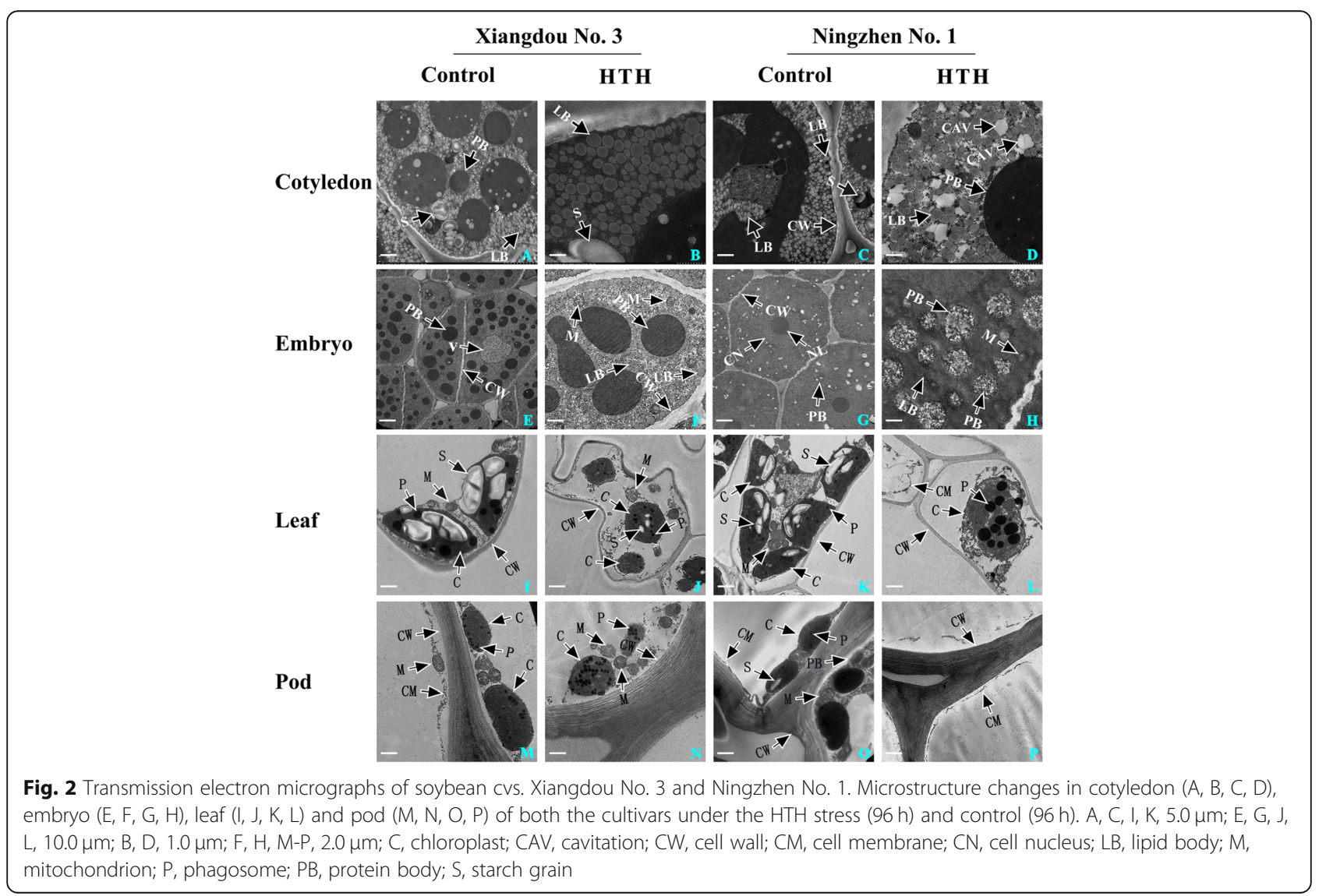


the soybean cultivars under the HTH stress, and furthermore, more severe decrease was found in soybean cv. Ningzhen No. 1 than in cv. Xiangdou No. 3 (Fig. 2I and J, Fig. $2 \mathrm{~K}$ and $\mathrm{L}$ ). In addition, a large number of phagosomes were observed in cv. Ningzhen No. 1 under the HTH stress, indicating that the senescence of its leaf cell was accelerated by stress (Fig. $2 \mathrm{~K}$ and L). In the pod (skin) cells, compared to the control (Fig. 2M), the number of mitochondrion in cv. Xiangdou No. 3 was increased after HTH stress (Fig. 2N). Interestingly, compared to the control (Fig. 2O), all the organelles in the pod (skin) cells of cv. Ningzhen No. 1 were disappeared under the HTH stress (Fig. 2P). All these results indicated that the HTH stress exerts greater negative effects on the leaf, pod, cotyledon and embryo of soybean cv. Ningzhen No. 1 than on those of cv. Xiangdou No. 3.

\section{Change of soluble protein, soluble sugar, malondialdehyde (MDA), starch contents under HTH stress}

The contents of soluble protein, soluble sugar, MDA and starch in the cotyledon, leaf, and pod (skin) of both the soybean cultivars were investigated, respectively (Fig. 3). The contents of starch (Fig. 3A and B) and sucrose (Fig. $3 \mathrm{C}$ and $\mathrm{D}$ ) were decreased in the cotyledon of both the cultivars under HTH stress, but the starch content of cv. Xiangdou No. 3 maintained higher level than that of cv. Ningzhen No. 1 at the stress time point of $168 \mathrm{~h}$. The soluble protein content in the cotyledon was decreased in cv. Ningzhen No. 1 but increased in cv. Xiangdou No. 3 under the HTH stress (Fig. 3E and F). In addition, the MDA content was increased in the cotyledon of both the cultivars under the HTH stress, but the MDA content in the cotyledon of cv. Ningzhen No. 1 maintained much higher level than in that of $\mathrm{cv}$. Xiangdou No. 3 (Fig. 3G and H). The HTH stress caused significant decrease in the starch (Fig. 3I and J, Fig. 3Q and R), sucrose (Fig. 3K and L, Fig. $3 \mathrm{~S}$ and T) and soluble protein (Fig. 3M and N, Fig. $3 \mathrm{U}$ and V) contents in the leaf and pod of both the soybean cultivars. However, the starch (Fig. 3J and R) and soluble protein (Fig. 3N and $\mathrm{V}$ ) contents in the leaf and pod, and the sucrose (Fig. 3L and T) contents in the pod of cv. Xiangdou No. 3 maintained higher level than those of cv. Ningzhen No. 1 at the stress time point of $168 \mathrm{~h}$. The contents of MDA were found to be increased in the leaf and pod of both the soybean cultivars under the HTH stress, but the MDA contents of cv. Ningzhen No. 1 were higher than those of cv. Xiangdou No. 3 (Fig. $3 \mathrm{O}$ and P, Fig. $3 \mathrm{~W}$ and $\mathrm{X}$ ).

The enzyme activities of almost all peroxidase (POD), superoxide dismutase (SOD) and catalase (CAT) in the cotyledon, embryo, leaf, and pod of cv. Xiangdou No. 3 were found to be significantly $(p<0.05$ or $p<0.01)$ increased under the HTH stress. However, the activities of most the enzymes were significantly $(p<0.05$ or $p<$ 0.01 ) reduced in cv. Ningzhen No. 1 under the HTH stress (Additional file 1: Fig. S1; Additional file 2: Fig. S2; Additional file 3: Fig. S3; Additional file 4: Fig. S4). These results indicated that the reactive oxygen species (ROS) scavenging capacity of soybean cv. Xiangdou No. 3 was stronger than that of cv. Ningzhen No. 1 under the HTH stress.

\section{Effect of HTH stress on germination of soybean seeds}

Compared to the controls, the germination potential, germination rate and seedling height of soybean cv. Xiangdou No. 3 were not significantly $(p>0.05)$ changed at the HTH stress time points of 24 and $96 \mathrm{~h}$, but markedly $(p<0.01)$ reduced at $168 \mathrm{~h}$ (Fig. $4 \mathrm{a}, \mathrm{c}$ and e). The germination potential and germination rate of soybean cv. Ningzhen No. 1 were significantly $(p<0.01)$ decreased at the stress time points of 96 and $168 \mathrm{~h}$ (Fig. 4b and d). The seedling height of soybean cv. Ningzhen No. 1 was significantly $(p<0.01)$ decreased at the stress time points of 24, 96 and $168 \mathrm{~h}$ (Fig. 4f). Moreover, the germination potential, germination rate, and seedling height of soybean cv. Ningzhen No. 1 were more significantly $(p<0.01)$ decreased than those of cv. Xiangdou No. 3 under the HTH stress (Fig. 4g, h and i). These results showed that soybean cv. Xiangdou No. 3 possessed higher seed germination vigor than cv. Ningzhen No. 1 under the HTH stress.

\section{Quantitative proteomic analysis in cotyledon, embryo, and leaf under HTH stress}

All the above results indicated that soybean cv. Xiangdou No. 3 was more tolerant to HTH stress than cv. Ningzhen No. 1 during seed growth and development. Therefore, the cotyledons, embryos and leaves from the stressed and control plants (R7 period) were sampled at 24,96 , and $168 \mathrm{~h}$ during the treatment, respectively, for iTRAQ analysis. In soybean cv. Ningzhen No. 1, a total of 235, 366 and 479 DAPs were identified in cotyledon, embryo and leaf under the $\mathrm{HTH}$ stress, respectively. Among them, 146 proteins in cotyledon, 120 proteins in embryo, and 235 proteins in leaf were found to be accumulated in abundance, whereas 89 proteins in cotyledon, 246 proteins in embryo, and 244 proteins in leaf were reduced in abundance (Fig. 5a; Additional file 5: S1-S3).

In soybean cv. Xiangdou No. 3, a total of 247, 179, and 517 DAPs were identified in cotyledon, embryo and leaf under the HTH stress, respectively. Among them, 134 proteins in cotyledon, 103 proteins in embryo, and 313 proteins in leaf were found to be accumulated in abundance, while 113 proteins in cotyledon, 76 proteins in embryo, and 204 proteins in leaf were reduced in abundance (Fig. 5b; Additional file 5: S4-S6). 


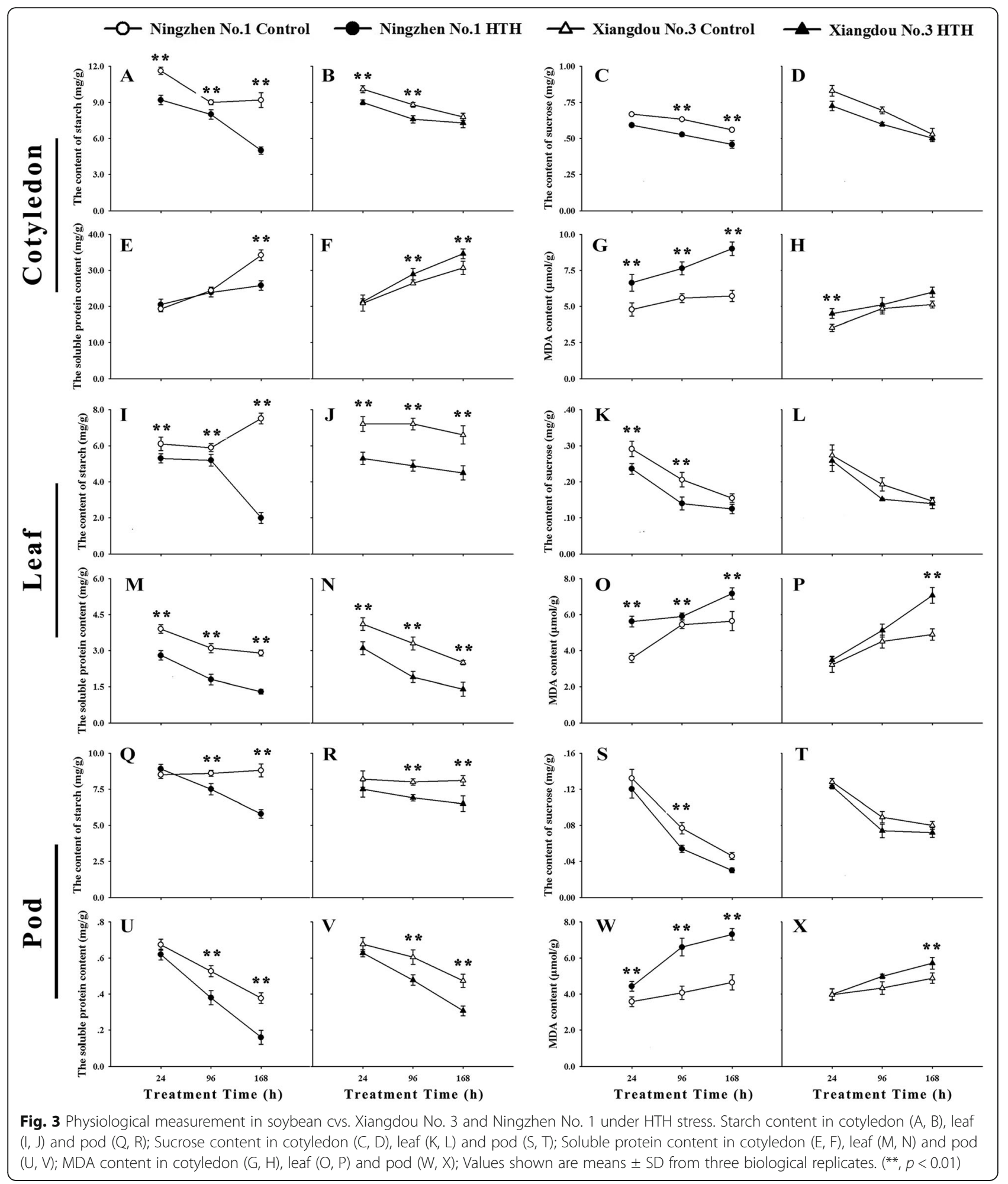

The proteome profiles of corresponding organs and HTH stress points were compared between the soybean cv. Xiangdou No. 3 and cv. Ningzhen No. 1. A total of 120,144 , and 438 DAPs were identified in the cotyledon, embryo and leaf, respectively. Among them, the abundances of 87 proteins in cotyledon, 64 proteins in embryo, and 221 proteins in leaf were found to be accumulated. In addition, the abundances of 33 proteins in cotyledon, 80 proteins in embryo, and 217 proteins in leaf were reduced (Fig. 5c; Additional file 5: S7- S9). 

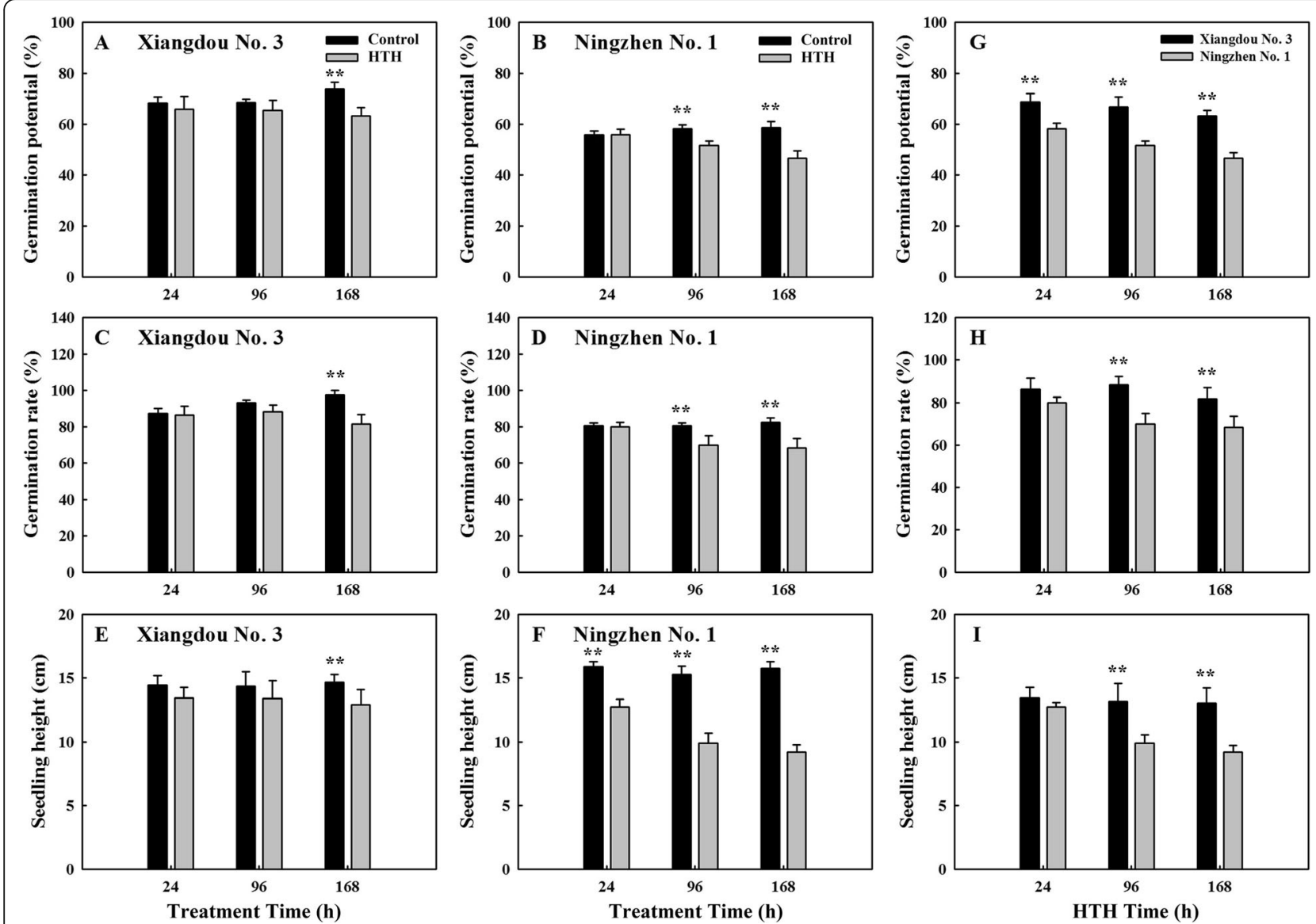

Fig. 4 Analysis of germination potential, germination rate and seedling height. The germination potential of pre-harvest seed deteriorationresistant soybean cv. Xiangdou No. 3 (a) and -sensitive cv. Ningzhen No. 1 (b) under the HTH stress and control. The germination rate of cv. Xiangdou No. 3 (c) and cv. Ningzhen No. 1 (d) under the HTH stress and control. The seedling height of cv. Xiangdou No. 3 (e) and cv. Ningzhen No. 1 (f) under the HTH stress and control. The germination potential (g), germination rate (h) and seedling height (i) of cv. Xiangdou No. 3 and cv. Ningzhen No. 1 under the HTH stress. Values are means \pm SD from three biological replicates $\left(^{* *}, p<0.01\right)$

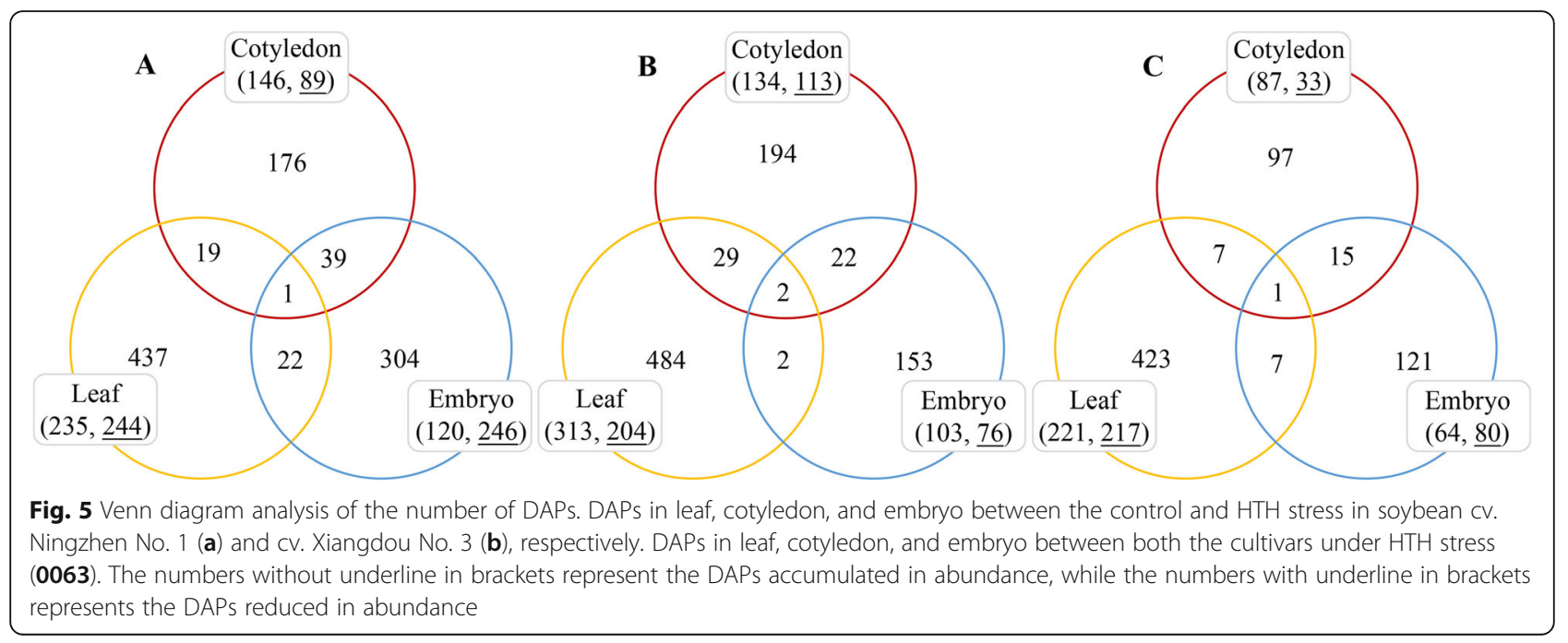




\section{GO and KEGG analysis of the DAPs under HTH stress}

The identified DAPs were further subjected to GO classification and KEGG pathway analysis. There were 42 metabolic pathways in the cotyledon and 50 in embryo altered in the pre-harvest seed deterioration-sensitive soybean cv. Ningzhen No. 1, while 37 in the cotyledon and 41 in the embryo were changed in the pre-harvest seed deterioration-resistant cv. Xiangdou No. 3 under HTH stress. Among them, the metabolic process, cytoplasm and enzyme regulator activity were the largest groups in the biological process, cellular component and molecular function categories, respectively, in the cotyledon and embryo of cv. Ningzhen No. 1 and in the embryo of cv. Xiangdou No. 3 (Additional file 7: Fig. S7 A; Additional file 8: Fig. S8 AB; Additional file 6: S1, S2, S5). The biosynthesis of terpenoids was enhanced, whereas the sucrose and starch metabolism was reduced in the cotyledon and embryo of cv. Ningzhen No. 1 (Additional file 6: S1, S2). In the cotyledon of cv. Xiangdou No. 3, the response to stimulus, cytoplasm and binding were the largest group in the biological process, cellular component and molecular function categories, respectively (Additional file 7: Fig. S7 B; Additional file 6: S4). The arginine biosynthesis was enhanced in the cotyledon and embryo of cv. Xiangdou No. 3 (Additional file 6: S4, S5). There were 53 and 57 metabolic pathways altered in the leaf of cvs Ningzhen No. 1 and Xiangdou No. 3, respectively. Among them, the metabolic process, chloroplast and binding were ranked the first in the biological process, cellular component and molecular function categories, respectively, in cv. Ningzhen No. 1 (Additional file 9: Fig. S9 A; Additional file 6: S3), whereas the metabolic process, chloroplast and enzyme regulator activity were ranked the first in the biological process, cellular component and molecular function categories, respectively, in cv. Xiangdou No. 3 (Additional file 9: Fig. S9 B; Additional file 6: S6). The protein processing in endoplasmic reticulum, tricarboxylic acid (TCA) cycle and fatty acid degradation were enhanced, while the photosynthesis, signal transduction and plant pathogen interaction were reduced in the leaf of cv. Ningzhen No. 1 (Additional file 6: S3). The TCA cycle, pyruvate metabolism and sulfur metabolism were enhanced, whereas the photosynthesis and plant pathogen interaction were reduced in the leaf of cv. Xiangdou No. 3 (Additional file 6: S6).

For between soybean cv. Xiangdou No. 3 and cv. Ningzhen No. 1 under the HTH stress, there were 26 and 29 metabolic pathways altered in the cotyledon and embryo, respectively. Among them, in cotyledon, the metabolic process, chloroplast and enzyme regulator activity were the largest groups in the biological process, cellular component and molecular function categories, respectively (Additional file 7: Fig. S7 C; Additional file
6: S7); in the embryo, the translation, cytoplasm and nutrient reservoir activity were the largest groups in the biological process, cellular component and molecular function categories, respectively (Additional file 8: Fig. S8 C; Additional file 6: S8). Moreover, in the cotyledon, the abscisic acid signaling, cytoskeleton, protein biosynthesis and oxidative stress response were enhanced under the $\mathrm{HTH}$ stress (Additional file 6: S7), while in the embryo, the abscisic acid signaling and oxidative stress response were enhanced under the HTH stress (Additional file 6: S8). There were 62 metabolic pathways found to be altered in the leaf. Among them, the metabolic process, chloroplast and enzyme regulator activity were ranked the first in the biological process, cellular component and molecular function categories, respectively (Additional file 9: Fig. S9 C; Additional file 6: S9). In addition, the arginine biosynthesis, carbon fixation, photosynthesis, TCA cycle, glutathione metabolism, and sulfur metabolism were enhanced, while the fatty acid degradation and phosphatidylinositol signaling were reduced (Additional file 6: S9).

\section{Transcriptional analysis of DAPs}

The quantitative RT-PCR (qRT-PCR) analysis was used to validate the expressions of eight genes encoding candidate HTH-responsive proteins identified in the developing seeds between soybean cv. Xiangdou No. 3 and cv. Ningzhen No. 1 under HTH stress by iTRAQ analysis. Six genes were found to be increased consistently at both the mRNA and protein levels under HTH stress in soybean cv. Xiangdou No. 3 compared to cv. Ningzhen No. 1. These six genes encode annexin (ANN), small heat shock protein (SHSP), SOD, dehydrin (DHD), stress-induced protein SAM22 (SAM), and camodulin (CAM), respectively. Besides, two genes encoding POD and lipoxygenase (LPX) showed inconsistency between the mRNA and protein levels (Fig. 6). This discrepancy might be attributed to posttranscriptional and posttranslational regulatory processes [26].

\section{Discussion}

High temperature and humidity are two pivotal factors that result in the decrease of soybean seed vigor during seed growth and development in the field, which is common in many soybean production areas around the world [6-8]. Recently, some studies have focused on the effects of HTH stress on the vigor formation of soybean developing seed using proteomic technologies [21-23], however, all these studies used the whole developing seed as experimental materials. In the present study, a comprehensive investigation was performed to reveal the effects of HTH stress on soybean seed vigor formation at levels of proteins, ultrastructure, and physiology and biochemistry using cotyledon, embryo, leaf, and pod. 


\section{口Q-PCR —ITRAQ}

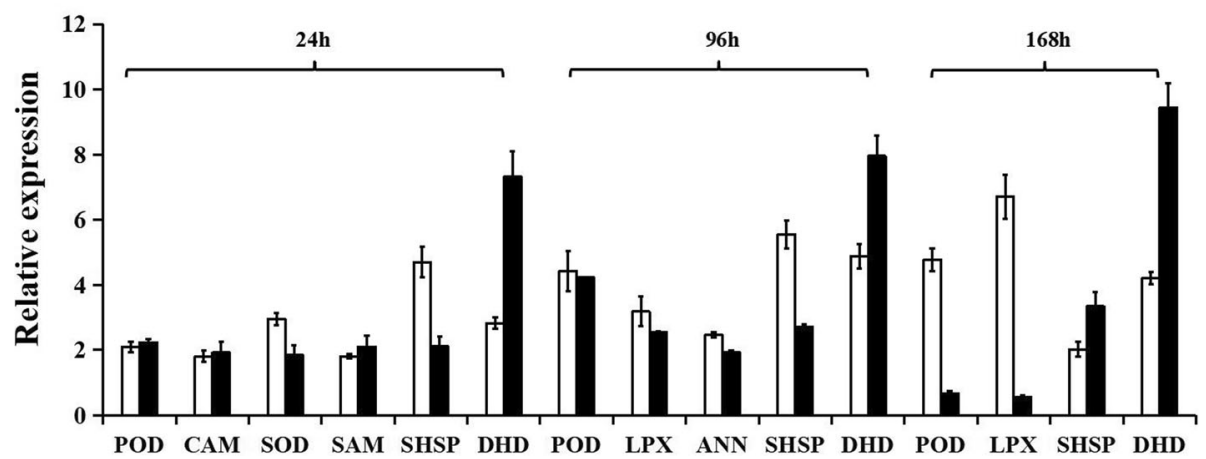

Fig. 6 Comparative analysis of mRNA and protein levels of selected genes encoding the differentially abundant proteins identified in seeds between soybean cv. Xiangdou No. 3 and cv. Ningzhen No. 1 under HTH stress. Values are means \pm SD $(n=3)$ through comparison of cv. Xiangdou No. 3 to cv. Ningzhen No. 1. Annexin, ANN; Calmodulin, CMD; Dehydrin, DHD; Lipoxygenase, LPX; Peroxidase, POD; Stress-induced protein SAM22, SAM; Superoxide dismutase, SOD; Small heat shock protein, chloroplastic, SHSP

\section{Comparison of effects of HTH stress on seed vigor} formation between the two soybean cultivars

In the cotyledon, the signal pathways [abscisic acid (ABA)mediated, $\mathrm{Ca}^{2+}$-mediated, and $\mathrm{G}$ protein-mediated], carbon fixation [ribulose bisphosphate carboxylase small chain $(\mathrm{Ru}-$ BisCO)], glycolysis [glyceraldehyde-3-phosphate dehydrogenase (GAPDH), alcohol dehydrogenase $(\mathrm{ADH})]$, cysteine and methionine biosynthesis [5-methyltetrahydropteroyltriglutamate- homocysteine methyltransferase (MT), methionine synthase (MS)], protein biosynthesis [40S ribosomal protein (RP), 60S RP], protein processing [heat shock protein STI (STI), SHSPs, SMP], and protein folding and assembly [chaperone protein (CPs), HSP 90] were enhanced, while the fatty acid degradation (polyunsaturated oxidation) was decreased only in soybean cv. Xiangdou No. 3 compared to cv. Ningzhen No. 1 under the HTH stress (Fig. 7; Additional file 10: Table S10). The increased protein biosynthesis in the cotyledon of soybean cv. Xiangdou No. 3 under the HTH stress was consistent with the changes of soluble protein contents (Fig. 7). Yin et al. [18] reported that ABA is involved in seed aging. Similar results in this study indicated that soybean cv. Xiangdou No. 3 may resist HTH stress by enhancing ABA signaling that may be caused by elevating endogenous ABA levels. RuBisCO is a key enzyme in Calvin cycle and plays a central role in the carbon fixation of photosynthesis. It catalyzes the carboxylation of ribulose-1,5-bisphosphate to yield two molecules of 3-phosphoglycerate, and simultaneously oxidizes the pentose substrate in the photorespiration process [27]. It has been reported that soybean seed at physiological maturity stage contains some mature chloroplasts without any photosynthesis activity [21]. Therefore, the accumulated $\mathrm{RuBisCO}$ implies that the photorespiration rate might be raised in the cotyledon of soybean cv. Xiangdou No. 3 in response to the HTH stress. Hirokazu et al. [28] showed that a reduction in $\mathrm{ADH}$ reduced seedling viability and decreased sugar concentrations in the rice seed. Additionally, AtADH1 overexpressing plants accumulated higher levels of total soluble sugars and sucrose than WT plants under control and stress conditions [29]. In this study, the accumulated $\mathrm{ADH}$ was accompanied with high levels of soluble proteins, sucrose and starch (Fig. 3), implying that the effects of ADH on seed vigor might be directly related to the enhanced nutrient storage. MT and MS catalyze the terminal step of de novo biosynthesis of methionine, which is an intermediate in the biosynthesis of polyamines, ethylene and biotin [30]. Increased biotin synthesis proteins are crucial for cell membrane stability under HTH stress [21]. In addition, Yacoubi et al. [12] reported that accumulated MT increased in parallel seed vigor, which is consistent with our TEM analysis and proteomic results (Fig. 2; Fig. 7). It has been reported that RPs perform independent functions of protein biosynthesis and the amounts of HSPs and CPs are all correlated closely with seed vigor. Catusse et al. [11] reported that the GAPDH and RPs were correlated positively with seed vigor in sugarbeet. Wu et al. [31] suggested that the HSPs were more abundant in high vigor maize seeds. Rajjou et al. [10] showed that GAPDH was increased, while CPs were inhibited in the artificially aged Arabidopsis seeds. In this study, our results showed that high levels of CAT, POD, SOD activities and low level of MDA content in the cotyledon of soybean cv. Xiangdou No. 3 under the HTH stress (Additional file 1: Fig. S1; Fig. 3 ), indicating that the function of cotyledon especially in cell ultrastructure stability, nutrient storage, and protein biosynthesis in pre-harvest seed deterioration-resistant soybean cv. Xiangdou No. 3 was stronger than in pre-harvest seed deterioration-sensitive soybean cv. Ningzhen No. 1 under HTH stress.

In the embryo, the signal pathways (G proteinmediated, and $\mathrm{Ca}^{2+}$-mediated, phosphatidylinositol), oxidative phosphorylation [Mitochondrial ATP synthase 


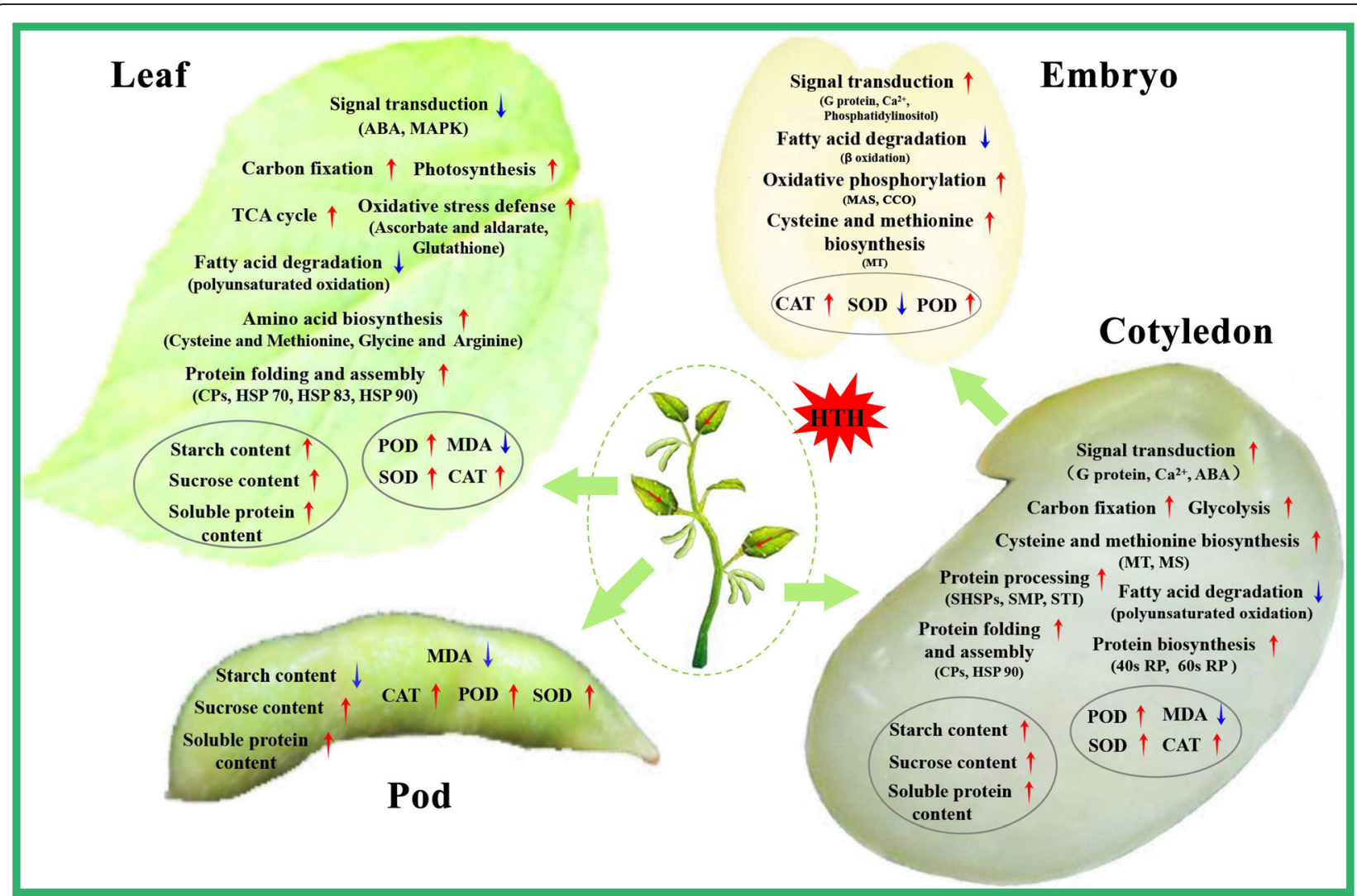

Fig. 7 Effects of HTH stress on physiological and biochemical processes in soybean. The major changed metabolic pathways, cellular processes and metabolites contents in leaf, pod skin, cotyledon, and embryo between the two cultivars under HTH stress. The positive metabolic pathways, cellular processes and metabolites are marked with " $\uparrow$ ", while the negativeis marked with " $\downarrow$ ". CAT, catalase; CP, chaperone protein; HTH, high temperature and humidity; MDA, malondialdehyde; MT, 5-methyltetrahydropteroyltriglutamate-homocysteine methyltransferase; MS, methionine synthase; POD, peroxidase; SHSP, small heat shock protein; SMP, seed maturation protein PM22; SOD, superoxide dismutase; STI, heat shock protein STI; RP, ribosomal protein
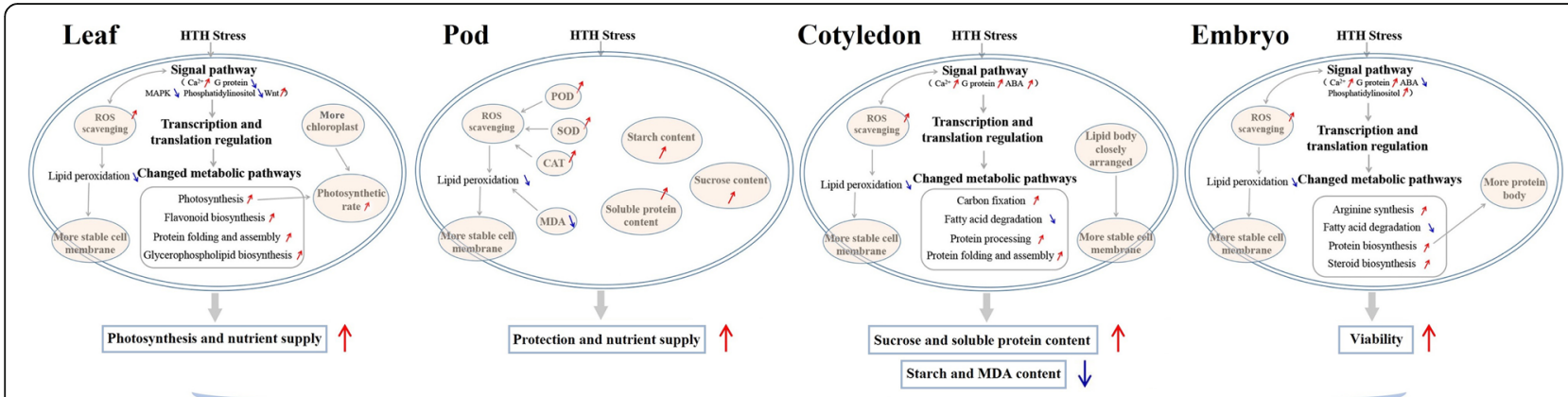

\section{Enhanced seed vigor}

Fig. 8 Graphic depiction for vigor enhancement mechanism in soybean under HTH stress. The enhanced metabolic pathways and cellular processes or the increased metabolites, in leaf, pod skin, cotyledon, and embryo are marked with " $\uparrow$ ", while the reduced marked with " $\downarrow$ ". ABA, abscisic acid; CAT, catalase; MAPK, mitogen-activated protein kinase; MDA, malondialdehyde; POD, peroxidase; ROS, reactive oxygen species; SOD, superoxide dismutase 
subunit O (MAS), cytochrome c oxidase (CCO)], and cysteine and methionine biosynthesis (MT) were enhanced and the ABA signaling and fatty acid degradation ( $\beta$ oxidation) were reduced only in soybean $\mathrm{cv}$. Xiagndou No. 3 compared to cv. Ningzhen No. 1 under the HTH stress (Fig. 7; Additional file 10: Table S10). ATP synthase subunit is a membrane-bound enzyme complexes transporter that combines ATP synthesis or hydrolysis with the transport of protons across the inner mitochondrial membrane [32]. CCO is the last complex IV of electron transfer chain in mitochondria and responsible for transferring electrons from oxidized cytochrome $\mathrm{c}$ to the final acceptor oxygen [33]. The accumulated MAS and CCO imply the increase of energy requirement during seed developing under HTH stress. Our previous seed viability test had proved that the soybean cv. Xiangdou No. 3 had stronger viability than cv. Ningzhen No. 1 under HTH stress [34]. Compared to a previous research [23], the phosphatidylinositol signaling was a novel finding, and it was increased in the embryo of soybean cv. Xiagndou No. 3 under the HTH stress in contrast to cv. Ningzhen No. 1. Phosphatidylinositol is a precursor structural phospholipid composed primarily of phosphorus, which can produce compounds involved in membrane integrity $[35,36]$. We speculated that the enhanced phosphatidylinositol signaling might maintain the stability of embryo cells in soybean cv. Xiagndou No. 3 under the HTH stress. Combined with the results of TEM, physiology and biochemistry (Fig. 2; Additional file 2: Fig. S2), our results indicated that the embryo of pre-harvest seed deterioration-resistant soybean cv. Xiangdou No. 3 had stronger viability than that of pre-harvest seed deterioration-sensitive soybean $\mathrm{cv}$. Ningzhen No. 1 under HTH stress.

The photosynthesis, carbon fixation, TCA cycle, oxidative stress defense, amino acid biosynthesis (cysteine, methionine, glycine, arginine), and protein folding and assembly (CPs, HSP 70, HSP 83, HSP 90) were enhanced, while the signaling $[\mathrm{ABA}$, mitogen-activated protein kinase (MAPK)] and fatty acid degradation (polyunsaturated oxidation) were reduced in the leaf of cv. Xiangdou No. 3, compared to that of cv. Ningzhen No. 1 under the HTH stress (Fig. 7; Additional file 10: Table S10). Here, the increased oxidative stress defense (ascorbate and aldarate, glutathione metabolism) in the leaf of soybean cv. Xiangdou No. 3 under the HTH stress compared to cv. Ningzhen No. 1 was consistent with the results of antioxidant enzymes activities (Fig. 7). ABA and MAPK play a pivotal role in transduction of diverse extracellular stimuli such as biotic and abiotic stresses as well as a range of developmental responses including differentiation, proliferation and death $[19,37]$. In this study, MAPK signaling was decreased in the leaf of soybean cv. Xiangdou No. 3 under the HTH stress, compared to soybean cv. Ningzhen No. 1, implying that cell death might be enhanced in the leaf of soybean cv. Ningzhen No. 1 under HTH the stress. Interestingly, the reduction of zeaxanthin epoxidase for ABA synthesis might suggest the existence of feedback regulation (Fig. 7; Additional file 6: Table S9). Consistent with proteomic results, the measured net photosynthetic rates were significantly $(p<0.01)$ higher in soybean cv. Xiangdou No. 3 than in cv. Ningzhen No. 1 under the HTH stress, which might attribute to more chloroplasts in the leaves of soybean cv. Xiangdou No. 3 (Fig. 1; Fig. 2). Beyond those, combined with high levels of starch and soluble protein contents in the leaves of soybean $\mathrm{cv}$. Xiangdou No. 3 under the HTH stress (Fig. 3), our results indicated that the leaf of pre-harvest seed deteriorationresistant soybean cv. Xiangdou No. 3 had stronger photosynthesis and nutrient supply than that of pre-harvest seed deterioration-sensitive soybean cv. Ningzhen No. 1 under HTH stress.

In the pod (skin) cell, TEM analysis showed that all the organelles disappeared in soybean cv. Ningzhen No. 1 under the HTH stress, while the number of mitochondria was increased in cv. Xiangdou No. 3 (Fig. 2). Mitochondria is a principal source of ROS in plant cells, and is the early target of oxidative injuries, which can be accelerated to a greater degree than in other organelles during deterioration [38,39]. The disappearance of organelles in the pod (skin) cell of soybean cv. Ningzhen No. 1 indicated that the cell ultrastructure was damaged under the HTH stress, whereas the cell ultrastructure in the pod (skin) cell of soybean cv. Xiangdou No. 3 was still maintained in a good condition. Moreover, a sharply increase in POD, CAT and SOD enzymes activities as well as high levels of starch, sucrose and soluble protein contents were found in the pod of soybean cv. Xiangdou No. 3 under the HTH stress (Fig. 3; Additional file 4: Fig. S4). These results indicated that the ROS scavenging and the nutrient transportation were enhanced in the pod of soybean cv. Xiangdou No. 3 under the HTH stress. In total, our results implied that the functions of pod in protection and nutrient supply for seeds in pre-harvest seed deterioration-resistant soybean cv. Xiangdou No.3 were still stronger than in preharvest seed deterioration-sensitive soybean cv. Ningzhen No. 1 under the HTH stress (Fig. 2; Fig. 3; Additional file 4: Fig. S4).

Taken together, when the seed (cotyledon and embryo), leaf, and pod of soybean were exposed to HTH stress, their signaling pathways (ABA-mediated, MAPK, G protein- mediated, $\mathrm{Ca}^{2+}$-mediated, and phosphatidylinositol) were severely affected. Thereafter, some metabolic pathways and cellular processes (photosynthesis, glycolysis, protein biosynthesis, protein folding and assembly, oxidative stress defense) were enhanced to improve seed vigor, while some other metabolic pathways and cellular processes would be lowered to reduce seed vigor. Under the persistent $\mathrm{HTH}$ stress, the relatively enhanced function of cotyledon (the 
increase of sucrose and soluble protein contents, the decrease of starch and MDA contents and protein biosynthesis), embryo (viability), leaf (photosynthesis and nutrient supply), and pod skin (protection and nutrients supply) led to the improvement of seed vigor (Fig. 8). The HTH stress had less negative effects on the signal pathways, metabolic pathways, cell ultrastructure, and physiology and biochemistry in the cotyledon, embryo, leaf, and pod of pre-harvest seed deterioration-resistant soybean cv. Xiangdou No. 3 than on those of pre-harvest seed deterioration-sensitive soybean cv. Ningzhen No. 1, leading to a higher seed vigor in Xiangdou No. 3 (Fig. 4). Song et al. [23] suggested that the developing seed of $\mathrm{cv}$. Xiangdou No. 3 possessed the greater ability of ROS scavenging, and cell rescue and defense than that of cv. Ningzhen No. 1 under HTH tress, which might be one of the major reasons why it was more deterioration-resistant than the latter. Thus, all these results will provide comprehensive characteristics of leaf, pod and seed under the HTH stress, which will be used as selection index in soybean breeding program for high seed vigor.

\section{Why the seeds produced from the HTH stressed plants could still germinate right?}

After $168 \mathrm{~h}$ of HTH stress, the plants of the two soybean cultivars was still alive, but the leaves were aged, and the photosynthetic capacity was basically lost (Fig. 1). Why the seeds produced from the stressed plants could still germinate right (Fig. 4)? The reasons might be as follows: firstly, the stressed time for the plants was chosen at physiological maturity (R7 period) when the seeds started to possess the capability of germination. The HTH stress of $168 \mathrm{~h}$ in the present study was incapable of causing seeds to completely lose the ability of germination. Secondly, when the leaves were being aged, their main nutrients were quickly transported to the seeds, guaranteeing seed development and maturity (Fig. 3). Thirdly, the pod would provide the protection for seeds. In addition, the seed itself had the mechanism of protection and repair under stress environment. However, there are great differences among soybean cultivars in resistance to $\mathrm{HTH}$ stress.

\section{The comparison between the HTH stress and the controlled deterioration treatment (CDT)}

$\mathrm{CDT}$ is widely used as a vigor assay for numerous seed species $[10,18,20]$. The harvested seeds are firstly subjected by CDT before the standard germination test. However, the HTH stress treatment in the present study is used to investigate its effects on the seed growth and development as well as germination at R7 period. Many studies have shown that CDT can affect the seed viability of Arabidopsis, Brassica napus, Oryza sativa and Glycine $\max [10,18,20,40]$. Some metabolic pathways were found to be changed in CDT and HTH treatments.
For example, HTH stress enhanced the accumulation of GAPDH in cotyledon of pre-harvest seed deteriorationresistant soybean cv. Xiangdou No. 3 (Fig. 7; Additional file 10: Table S10), whereas a decrease in GAPDH level was found in aged Arabidopsis and wheat seeds through CDT [10, 41]. Zhang et al. [19] reported that rice seed aging (through $\mathrm{CDT}$ ) was associated with increased abundance of $\mathrm{ADH}$ in the embryo, whereas the similar result was found in cotyledon between the two soybean cultivars under HTH stress in this study (Fig. 7). All the results of our research and Rajjou et al. [10] indicated that cysteine synthesis was an important feature of germination potential. Yin et al. [18] reported the involvement of $\mathrm{ABA}$ in the initiation of seed aging. Interestingly, in this study, compared to soybean cv. Ningzhen No. 1, the abscisic acid signaling was increased in cotyledon of cv. Xiangdou No. 3 under the HTH stress, but decreased in embryo.

\section{Conclusion}

The HTH stress affected seed vigor through the negative effects on the signal pathways (ABA-mediated, MAPK, G protein-mediated, $\mathrm{Ca}^{2+}$-mediated, and phosphatidylinositol), metabolic pathways (photosynthesis, glycolysis, protein biosynthesis, protein folding and assembly, oxidative stress defense), cell ultrastructure, and physiology and biochemistry (antioxidases activities, sucrose, starch and soluble protein contents) in leaf, pod, cotyledon, and embryo of soybean. Soybean cultivars more tolerant to HTH stress produce higher vigor seeds.

\section{Methods}

\section{Planting and sampling}

Pre-harvest seed deterioration-resistant cv. Xiangdou No. 3 and -sensitive cv. Ningzhen No. 1, which were previously screened out by incubator weathering followed by standard germination test, were used in this study [21]. Soybean cvs. Xiangdou No. 3 and Ningzhen No. 1 were two extension cultivars in South China. Soybean cv. Xiangdou No. 3 was bred by Hunan Academy of Agricultural Sciences, while Soybean cv. Ningzhen No. 1 was bred by Jiangsu Academy of Agricultural Sciences in China. Seedlings of the two cultivars were grown in plastic pots. The plants were divided into two groups when they reached the physiological maturity period (R7). The stressed group was evenly transferred to three independent growth chambers $\left[40^{\circ} \mathrm{C} / 30^{\circ} \mathrm{C}\right.$, $100 \% / 70 \%$ humidity, and $10 \mathrm{~h} / 14 \mathrm{~h}$ cycle (day/night)] for $7 \mathrm{~d}$. The control group with the same developmental progression were also placed in three separate chambers under $30^{\circ} \mathrm{C} / 20^{\circ} \mathrm{C}, 70 \%$ humidity, and $10 \mathrm{~h} / 14 \mathrm{~h}$ (day/ night). The seeds (cotyledons and embryos), leaves and pods in the middle of the plants (10 plants) were sampled at 24, 96, and $168 \mathrm{~h}$ from each chamber. Therefore, 
each treatment or control has three biological replicates. The embryos stripped carefully from sample seeds by blade. Collected samples were frozen in liquid nitrogen immediately and stored at $-80^{\circ} \mathrm{C}$ until use.

\section{Photosynthesis measurement}

Leaf net photosynthetic rates were measured using a portable gas analysis system, LI-COR 6400 (Li-Cor Inc., Lincoln, NE, USA) according to [42]. The measurement conditions were set as follows: leaf temperature at $25^{\circ} \mathrm{C}$, $1000 \mu \mathrm{mol}$ photons $\mathrm{m}^{-2} \cdot \mathrm{s}^{-1}$. Each sample was measured for three times. All measurements were carried out between 9:00 am and 11:00 am to minimize the error.

\section{Transmission electron microscope analysis}

TEM analysis was conducted according to [43]. Fresh leaves, pods, cotyledons and embryos from treatments and controls were cut into $2 \mathrm{~mm} \times 3 \mathrm{~mm}$, respectively, and fixed in a mixture of $2.5 \%$ glutaraldehyde in a 20 $\mathrm{mM}$ sodium cacodylate buffer $(\mathrm{pH} 7.0)$ for at least $12 \mathrm{~h}$ at $4{ }^{\circ} \mathrm{C}$, and then postfixed with $1 \% \mathrm{KMnO}_{4}$ for $2 \mathrm{~h}$. These fixed samples were dehydrated in an ethanol series and embedded in Spurr resin. Ultrathin sections (70 $\mathrm{nm}$ thick) were stained with uranyl acetate and lead citrate. Observations were made on an H-7650 (Tokyo, Japan) TEM. Construction of high-resolution TEM pictures was carried out as described by [44].

\section{Enzyme activity assays}

Soybean leaves, pods, cotyledons and embryos ( $0.5 \mathrm{~g})$ from treatments and controls was ground, respectively, in a mortar with $1 \mathrm{ml}$ of chilled $0.1 \mathrm{M}$ phosphate buffer ( $\mathrm{pH}$ 7.5) containing $1 \%(\mathrm{w} / \mathrm{v})$ polyvinylpolypyrrolidone. Homogenates were centrifuged at $14,000 \times g$ at $4{ }^{\circ} \mathrm{C}$ for $30 \mathrm{~min}$ and the supernatants were used for enzyme activity assays. Five technical replicates were performed for each sample. Enzyme activities of CAT, POD and SOD were determined following the procedure described by [45]. All experiments were repeated for three times.

\section{Contents analysis of soluble protein, soluble sugar, MDA and starch}

Soybean leaves, pods and cotyledons $(0.5 \mathrm{~g})$ from treatments and controls were used in this experiment. Concentration of the soluble protein was measured according to [46] with bovine serum albumin as standard. Content of soluble sugar was determined as previously described in [47]. The level of lipid peroxidation was determined as described in [48]. The total starch content was analyzed according to the method of [49]. All experiments were repeated for three times.

\section{Germination test}

A series of germination tests were performed at 24, 96 and $168 \mathrm{~h}$ after HTH stress, respectively, according to [50]. Three replications (50 seeds for each treatment) were distributed in plastic boxes $(20 \times 11.5 \times 8 \mathrm{~cm})$ containing six sheets of moistened filter paper. Plastic boxes were put in a germination chamber at $20^{\circ} \mathrm{C}, 60 \%$ humidity, $8 \mathrm{~h}$ light and $16 \mathrm{~h}$ darkness. Normal seedlings were recorded every day following incubation until 7 days, germination potential, germination rate and seedling height were determined, germination potential was assessed by the germination rate of the seeds on the fourth day.

\section{Protein extraction, digestion and labeling}

Protein extraction was conducted as reported by [51]. The protein concentration was determined by the method of Bradford [46] using bovine serum albumin as standard. For each sample, an aliquot $(100 \mu \mathrm{g})$ of protein was reduced with $10 \mathrm{mM}$ dithiothreitol for $1 \mathrm{~h}$ at $37^{\circ} \mathrm{C}$ and alkylated with $20 \mathrm{mM}$ iodoacetamide for $1 \mathrm{~h}$ at room temperature in dark. Protein samples were digested using an enzyme to substrate ratio of 1:20 sequencinggrade trypsin (Promega, Madison, WI) at $37^{\circ} \mathrm{C}$ overnight and then resultant peptide mixture was labeled using chemicals from iTRAQ reagent kit (Applied Biosystems, California, USA). All the iTRAQ labels were shown in Additional file 11: Table S11. The changes of protein level within cultivar at different time points were determined through comparison of point to point between the HTH treatment and the control in the same sevenplex. To compare the changes of protein level between the two cultivars under HTH stress, first, all the protein level data were normalized through comparison with the mixed sample from cv. Ningzhen No. 1 and cv. Xiangdou No. 3 at $24 \mathrm{~h}$ under the control condition in the same seven-plex; second, for each protein identified, its relative level ratios of cv. Xiangdou No. 3 to cv. Ningzhen No. 1 under the HTH stress were calculated as follows.

$$
\begin{aligned}
\text { Relative protein level ratio }= & \left(\mathrm{X}_{\mathrm{T}} \div \mathrm{N}_{\mathrm{T}}\right) \\
& \div\left(\mathrm{X}_{\mathrm{C}} \div \mathrm{N}_{\mathrm{C}}\right)
\end{aligned}
$$

Where, $\mathrm{X}_{\mathrm{T}}$ and $\mathrm{N}_{\mathrm{T}}$ indicate the relative level of a protein in cv. Xiangdou No. 3 and cv. Ningzhen No. 1 under HTH stress after normalization, respectively, while $\mathrm{X}_{\mathrm{C}}$ and $\mathrm{N}_{\mathrm{C}}$ in cv. Xiangdou No. 3 and cv. Ningzhen No. 1 under control condition after normalization, respectively.

\section{iTRAQ analysis}

For each organ sample (leaf, cotyledon, and embryo) that was equal weight mixed sample of the organ from 10 
plants, three iTRAQ biological repeats were conducted. Therefore, there were 27 groups ( 3 cultivars or cultivar combinations $\times 3$ organs $\times 3$ biological replicates) data in the present study (Additional file 11: Table S11). Each iTRAQ reagent was dissolved in $50 \mu \mathrm{l}$ of isopropanol, which was added to the respective peptide mixture. The digested peptides were labeled with iTRAQ reagents following the manufacturer's instructions (AB Sciex). Firstly, the peptides were fractionated on a waters UPLC using a high $\mathrm{pH}$ C18 column (Waters Bec C18, $1.7 \mu \mathrm{m}$, $2.1 \mathrm{~mm} \times 50 \mu \mathrm{m}$ ), then the fractionations were analyzed by nano-HPLC on the secondary reverse phase analytical column (Eksigent, C18, $3 \mu \mathrm{m}, 150 \mathrm{~mm} \times 75 \mu \mathrm{m}$ ). Peptides were eluted using a linear gradient, starting from 5 to $45 \%$ buffer B in 70 min (buffer A, $98 \%$ water with $0.1 \%$ formic acid, buffer B, $98 \%$ acetonitrile with $0.1 \%$ formic acid). The total flow rate was maintained at $300 \mathrm{~nL} / \mathrm{min}$. Electrospray voltage of $2.3 \mathrm{kV}$ versus the inlet of the mass spectrometer was used. TripleTOF 5600 mass spectrometer was operated in data-dependent mode to switch automatically between MS and MS/MS acquisition. MS spectra were acquired across the mass range of $350-1250 \mathrm{~m} / \mathrm{z}$ in high resolution mode using $250 \mathrm{~ms}$ accumulation time per spectrum. Tandem mass spectral scanned from 100 to $1250 \mathrm{~m} / \mathrm{z}$ in high sensitivity mode with rolling collision energy. The 20 most intense precursors were selected for fragmentation per cycle with dynamic exclusion time of $9 \mathrm{~s}$.

\section{Mass spectra data and protein quantification}

Maxquant software v. 1.5.2.8 was used for large-scale tandem mass spectrometry data analysis [52]. The Glycine max UniProtKB/Swiss-Prot database were used, and downloaded on December, 2016 with 124,278 sequences. The wiff files generated by the TripleTOF 5600 instrument were searched directly using a $20 \mathrm{ppm}$ precursor mass tolerance and a $50 \mathrm{mmu}$ fragment mass tolerance. All the peptides FDR were dynamically set as $1 \%$, which were calculated by a decoy database search (using a reverse sequence version of the reference database), and each confident protein included at least one unique peptide [53]. Redundancy between the annotated proteins and proteins identified from translation of the soybean genome was identified using BLAST [54], and all the identified proteins was list in Additional file 12: S1-S9. Screening criteria for DAPs were as follows: the proteins with a t-test $p$-value $<0.05$ for two compared groups with three replicates, peptides $\geq 2$ and a protein ratio $>$ 1.5 or $<0.67$-fold change [55].

\section{Bioinformatics analysis}

The functional analysis of DAPs was conducted using Gene Ontology (GO) (http://www.ebi.ac.uk/QuickGO/) [56]. The DAPs were further assigned by the KEGG
(Kyoto Encyclopedia of Genes and Genomes) database (http://www.kegg.jp/kegg/pathway.html) [57].

\section{Quantitative RT-PCR analysis}

The expressions of eight genes encoding candidate $\mathrm{HTH}$-responsive proteins were analyzed by qRT-PCR. These HTH-responsive proteins were differentially accumulated in the seeds between soybean cv. Xiangdou No. 3 and cv. Ningzhen No. 1. Total RNA was extracted from seeds, and then qRT-PCR was performed according to protocol of Liu et al. [58]. The primers were listed in Additional file 13: Table S13. The relative quantification $\left(2^{-\Delta \Delta \mathrm{Ct}}\right)$ of gene expression was evaluated using comparative cycle threshold method, and each sample was replicated for three times.

\section{Statistical analysis}

The $t$-test was used for pair-wise comparison of proteomic data and analysis of the significant changes in physiological data (SPSS 19.0, IBM, USA) with a confidence interval of $95 \%$ or $99 \%$.

\section{Supplementary information}

Supplementary information accompanies this paper at https://doi.org/10. 1186/s12870-020-02335-1.

Additional file 1 : Figure S1. Enzyme activities of CAT, POD, and SOD in the cotyledons of soybean cvs. Xiangdou No. 3 and Ningzhen No. 1

Additional file 2 : Figure S2. Enzyme activities of CAT, POD, and SOD in the embryos of soybean cvs. Xiangdou No. 3 and Ningzhen No. 1

Additional file 3 : Figure S3. Enzyme activities of CAT, POD, and SOD in the leaves of soybean cvs. Xiangdou No. 3 and Ningzhen No. 1

Additional file 4 : Figure S4. Enzyme activities of CAT, POD, and SOD in the pod skin of soybean cvs. Xiangdou No. 3 and Ningzhen No. 1

Additional file $\mathbf{5}$ : $\mathbf{S 1 -} \mathbf{S 6}$. Differentially abundant proteins in cotyledons, embryos, and leaves between the stressed and control in soybean cvs. Ningzhen No. 1 and Xiangdou No. 3, respectively. S7- S9. Differentially abundant proteins in cotyledons, embryos, and leaves between the soybean cv. Ningzhen No. 1 and cv. Xiangdou No. 3 under HTH stress

Additional file 6 : S1- S9. GO and KEGG analysis of the differentially abundant proteins in cotyledons, embryos, and leaves

Additional file $\mathbf{7}$ : Figure S7. Go classification of differentially abundant proteins identified in cotyledons between the control and the stressed in soybean cvs. Ningzhen No. 1 (A) and Xiangdou No. 3 (B), respectively, and between both the cultivars under the HTH stress (C)

Additional file 8 : Figure $\mathbf{S 8}$. Go classification of differentially abundant proteins identified in embryos between the control and the stressed in soybean cvs. Ningzhen No. 1 (A) and Xiangdou No. 3 (B), respectively, and between both the cultivars under the HTH stress (C)

Additional file 9 : Figure S9. Go classification of differentially abundant proteins identified in leaves between the control and the stressed in soybean cvs. Ningzhen No. 1 (A) and Xiangdou No. 3 (B), respectively, and between both the cultivars under the HTH stress (C)

Additional file 10 : Table S10. Key KEGG pathways for seed vigor formation in leaves, cotyledons, and embryos between the stressed and control in soybean cvs. Ningzhen No. 1 and Xiangdou No. 3, respectively, and between both the cultivars

Additional file 11 : Table S11. 7-plex ITRAQ experimental design 
Additional file 12 : S1-S9. The list of all the identified proteins Additional file 13 : Table S13. Sequences of primers used in this study

\section{Abbreviations}

ABA: Abscisic acid; ADH: Alcohol dehydrogenase; CAT: Catalase; CCO: Cytochrome c oxidase; CDT: Controlled deterioration treatment; CP: Chaperone protein; CV: Cultivar; DAP: Differentially abundant protein; DHD: Dehydrin; GAPDH: Glyceraldehyde-3-phosphate dehydrogenase; GO: Gene Ontology; HSP: Heat shock protein; HTH: High temperature and humidity; iTRAQ: Isobaric tags for relative and absolute quantification; KEGG: Kyoto Encyclopedia of Genes and Genomes; LPX: Lipoxygenase; MAPK: Mitogen-activated protein kinase; MDA: Malondialdehyde; MAS: Mitochondrial ATP synthase subunit O; MT: 5 methyltetrahydropteroyltriglutamate--homocysteine methyltransferase; MS: Methionine synthase; POD: Peroxidase; ROS: Reactive oxygen species; RP: Ribosomal protein; RuBisCO: Ribulose bisphosphate carboxylase small chain; SHSP: Small heat shock protein; SOD: Superoxide dismutase; STI: Heat shock protein STI; TCA: Tricarboxylic acid; TEM: Transmission electron microscope

\section{Acknowledgements}

The authors are thankful to Xingwang Yu, Crop and Soil Sciences Department, North Carolina State University, United States for polishing language of this paper.

\section{Authors' contributions}

The work presented here was carried out in collaboration among all authors. J.W. carried out most of the laboratory experiments; YJ.S., X.L. and YL.Z. carried out the seed vigor assay; YZ.S., S.L. and H.Z. carried out the protein data analysis; L.L. and YJ.Z. carried out the enzyme activities assay. H.M. designed the experiments and wrote the manuscript. $X$. $Y$ revised and polished the manuscript. All authors have read and approved the final manuscript.

\section{Funding}

The project was supported by the National Natural Science Foundation of China (31101212, 30971840, 31171572, 31371711, 31671772, and 31971996), the Ministry of Science and Technology of China (2018YFD0100905), and the Cyrus Tang Innovation Center for Seed Industry for this research.

\section{Availability of data and materials}

The data sets supporting the conclusions of this article are included within the article and its additional files. The mass spectrometry proteomic data have been submitted to a public iProX database (https://www.iprox.org/ page/PSV023.html;?url=1578575601558rHFc), the password is I9ux.

\section{Ethics approval and consent to participate}

Not applicable.

\section{Consent for publication}

Not applicable.

\section{Competing interests}

The authors declare that they have no competing interests.

\section{Author details \\ 'State Key Laboratory of Crop Genetics and Germplasm Enhancement, Nanjing Agricultural University, Nanjing 210095, China. ${ }^{2}$ Crop and Soil Sciences Department, North Carolina State University, Raleigh, NC 27695, USA. ${ }^{3}$ College of Agriculture, Anhui Science and Technology University, Fengyang 233100, China.}

\section{Received: 1 February 2019 Accepted: 9 March 2020}

Published online: 26 March 2020

\section{References}

1. Zhang GY, Bahn SC, Wang GL, Zhang YR, Chen BB, Zhang YL, Wang XM, Zhao J. PLDa1-knockdown soybean seeds display higher unsaturated glycerolipid contents and seed vigor in high temperature and humidity environments. Biotechnol Biofuels. 2019;12:9.
2. Ray DK, Gerber JS, MacDonald GK, West PC. Climate variation explains a third of global crop yield variability. Nat Commun. 2015;6:5989.

3. Shu YJ, Tao Y, Wang S, Huang LY, Yu XW, Wang ZK, Chen M, Gu WH, Ma H. $\mathrm{GmSBH1}$, a homeobox transcription factor gene, related to growth and development and involves in response to high temperature and humidity stress in soybean. Plant Cell Rep. 2015;34:1927-37.

4. Narayanan S, Tamura PJ, Roth MR, Prasad PV, Welti R. Wheat leaf lipids during heat stress: I. high day and night temperatures result in major lipid alterations. Plant Cell Environ. 2016:39:787-803.

5. Hossain Z, Khatoon A, Komatsu S. Soybean proteomics for unraveling abiotic stress response mechanism. J Proteome Res. 2013;12(11):4670-84.

6. Egli DB, TeKrony DM, Heitholt JJ, Rupe J. Air temperature during seed filling and soybean seed germination and vigor. Crop Sci. 2005;45:1329-35.

7. Wang F, Wang LQ, Tian X, Gu WH, Ma H. Pre-harvest and post-harvest seed deterioration resistance of spring soybean germplasm in South China. Chin Agric Sci. 2007:40:2637-47

8. Ren C, Bilyeu KD, Beuselinck PR. Composition, vigor, and proteome of mature soybean seeds developed under high temperature. Crop Sci. 2009; 49:1010-22.

9. Wang WQ, Liu SJ, Song SQ, Moller IM. Proteomics of seed development, desiccation tolerance, germination and vigor. Plant Physiol Biochem. 2015; $86: 1-15$.

10. Raijou L, Lovigny Y, Groot SPC, Belghazi M, Job C, Job D. Proteome-wide characterization of seed aging in Arabidopsis: a comparison between artificial and natural aging protocols. Plant Physiol. 2008;148(1):620-41.

11. Catusse J, Meinhard J, Job C, Strub JM, Fischer U, Pestsova E, Westhoff P, Dorsselaer AV, Job D. Proteomics reveals potential biomarkers of seed vigor in sugarbeet. Proteomics. 2011;11(9):1569-80.

12. Yacoubi R, Job C, Belghazi M, Chaibi W, Job D. Toward characterizing seed vigor in alfalfa through proteomic analysis of germination and priming. J Proteome Res. 2011;10(9):3891-903

13. Xin $X$, Lin $X H$, Zhou YC, Chen XL, Liu X, Lu XX. Proteome analysis of maize seeds: the effect of artificial ageing. Plant Physiol. 2011;143(2):126-38.

14. Yao Z, Liu L, Gao F, Rampitsch C, Reinecke DM, Ozga JA, Ayele BT. Developmental and seed aging mediated regulation of antioxidative genes and differential expression of proteins during pre- and post-germinative phases in pea. J Plant Physiol. 2012;169(15):1477-88.

15. Raijou L, Duval M, Gallardo K, Catusse J, Bally J, Job C, Job D. Seed germination and vigor. Plant Biol. 2012;63:507-33.

16. Chu P, Chen HH, Zhou YL, Li Y, Ding Y, Jiang LW, Tsang EWT, Wu K, Huang SZ. Proteomic and functional analyses of nelumbo nucifera annexins involved in seed thermotolerance and germination vigor. Planta. 2012; 235(6):1271-88.

17. Zhang H, Wang WQ, Liu SJ, Moller IM, Song SQ. Proteome analysis of poplar seed vigor. PLoS One. 2015;10(7):e0132509.

18. Yin X, He D, Gupta R, Yang P. Physiological and proteomic analyses on artificially aged Brassica napus seed. Front Plant Sci. 2015:6:112

19. Zhang YX, Xu HH, Liu SJ, Li N, Wang WQ, Moller IM, Song SQ. Proteomic analysis reveals different involvement of embryo and endosperm proteins during aging of Yliangyou 2 hybrid rice seeds. Front Plant Sci. 2016;7:1394

20. Min CW, Lee SH, Cheon YE, Han WY, Ko JM, Kang HW, Kim YC, Agrawal GK, Rakwal R, Gupta R. In-depth proteomic analysis of Glycine max seeds during controlled deterioration treatment reveals a shift in seed metabolism. J Proteome. 2017;169:125-35.

21. Wang LQ, Ma H, Song LR, Shu YJ, Gu WH. Comparative proteomics analysis reveals the mechanism of pre-harvest seed deterioration of soybean under high temperature and humidity stress. J Proteome. 2012;75(7):2109-27.

22. Ma H, Wang LQ, Wang S, Wei JP, Huang LY, Gu WH. Comparative proteomics analysis of developing seed of a pre-harvest seed deterioration resistant soybean cultivar under high temperature and humidity stress. Curr Proteomics. 2015;12:168-84.

23. Song LR, Wang S, Niu J, Ma HY, Shu YJ, Yang Y, Gu WH, Ma H. Differentially proteomics analysis of pre-harvest seed deterioration and deterioration resistance in spring soybean. Chin Agric Sci. 2015:48:23-32.

24. Evans JR, von Caemmerer S. Enhancing photosynthesis. Plant Physiol. 2011; 155:19.

25. Makino A. Photosynthesis, grain yield, and nitrogen utilization in rice and wheat. Plant Physiol. 2011:155(1):125-9.

26. Yang LT, Qi YP, Lu YB, Guo P, Sang W, Feng H, Zhang HX, Chen LS. iTRAQ protein profile analysis of Citrus sinensis roots in response to long-term boron-deficiency. J Proteome. 2013;93:179-206. 
27. Matsumoto K, Saito J, Yokoo T, Hori C, Nagata A. Ribulose-1,5-bisphosphate carboxylase/oxygenase (RuBisCO)-mediated de novo synthesis of glycolatebased polyhydroxyalkanoate in Escherichia coli. J Biosci Bioeng. 2019;128: 302-6.

28. Takahashi H, Greenway H, Matsumura H, Tsutsumi N, Nakazono M. Rice alcohol dehydrogenase 1 promotes survival and has a major impact on carbohydrate metabolism in the embryo and endosperm when seeds are germinated in partially oxygenated water. Ann Bot. 2014;113:851-9.

29. Shi H, Liu W, Yao Y, Wei Y, Chan Z. Alcohol dehydrogenase 1 (ADH1) confers both abiotic and biotic stress resistance in Arabidopsis. Plant Sci. 2017;262:24-31.

30. Gomez-Garay A, Lopez JA, Camafeita E, Bueno MA, Pintos B. Proteomic perspective of Quercus suber somatic embryogenesis. J Proteome. 2013;93: 314-25.

31. Wu X, Liu H, Wang W, Chen S, Hu X, Li C. Proteomic analysis of seed viability in maize. Acta Physiol Plant. 2011;33:181-91.

32. Klusch N, Murphy BJ, Mills DJ, Yildiz O, Kuhlbrandt W. Structural basis of proton translocation and force generation in mitochondrial ATP synthase. Elife. 2017;6:e33274.

33. Dahan J, Tcherkez G, Macherel D, Benamar A, Belcram K. Disruption of the CYTOCHROME C OXIDASE DEFICIENT1 gene leads to cytochrome c oxidase depletion and reorchestrated respiratory metabolism in Arabidopsis. Plant Physiol. 2014;166:1788-802.

34. Shu YJ. Isolation and expression analysis of GmSbh1 that involved in seed deterioration. Nanjing: PhD, Nanjing Agricultural University; 2014. p. 24-6.

35. Vermeer JEM, Thole JM, Goedhart J, Nielsen E, Munnik T, Gadella TW. Imaging phosphatidylinositol 4-phosphate dynamics in living plant cells. Plant J. 2009;57(2):356-72.

36. Ischebeck T, Stenzel I, Hempel F, Jin X, Mosblech A, Heilmann I. Phosphatidylinositol-4,5-bisphosphate influences Nt-Rac5-mediated cell expansion in pollen tubes of Nicotiana tabacum. Plant J. 2011;65(3):453-68

37. Taj $G$, Agarwal $P$, Grant $M$, Kumar A. MAPK machinery in plants recognition and response to different stresses through multiple signal transduction pathways. Plant Signal Behav. 2010;5(11):1370-8.

38. Gupta SD. Reactive oxygen species and antioxidants in higher plant. New York: CRC Press; 2011. p. 189

39. Jacoby RP, Li L, Huang S, Pong Lee C, Millar AH, Taylor NL. Mitochondrial composition, function and stress response in plants. J Integr Plant Biol. 2012:54(11):887-906

40. Gao J, Fu H, Zhou X, Chen Z, Luo Y, Cui B, Chen G, Liu J. Comparative proteomic analysis of seed embryo proteins associated with seed storability in rice (Oryza sativa L) during natural aging. Plant Physiol Biochem. 2016; 103:31-44.

41. Lv Y, Zhang S, Wang J, Hu Y. Quantitative proteomic analysis of wheat seeds during artificial ageing and priming using the isobaric tandem mass tag labeling. PLoS One. 2016;11(9):e0162851

42. Chu P, Yan GX, Yang Q, Zhai LN, Zhang C, Zhang FQ, Guan RZ. iTRAQ-based quantitative proteomics analysis of brassica napus leaves reveals pathways associated with chlorophyll deficiency. J Proteome. 2015:113:244-59.

43. Higaki T, Kutsuna N, Akita K, Sato M, Sawaki F, Kobayashi M, Nagata N, Toyooka K, Hasezawa S. Semi-automatic organelle detection on transmission electron microscopic images. Sci Rep. 2015;5:7794.

44. Toyooka K, Sato M, Kutsuna N, Nagata N. Development of high resolution TEM image acquisition system by using high-pressure freezing method. Plant Morphology. 2014;26:3-8.

45. Song LR, Liu ZQ, Tong JH, Xiao LT, Ma H, Zhang HQ. Comparative proteomics analysis reveals the mechanism of fertility alternation of thermosensitive genic male sterile rice lines under low temperature inducement. Proteomics. 2015;15(11):1884-905.

46. Bradford MM. A rapid and sensitive method for the quantitation of microgram quantities of protein utilizing the principle of protein-dye binding. Anal Biochem. 1976;72:248-54.

47. Cheng L, Wang Y, He Q, Li H, Zhang X, Zhang F. Comparative proteomics illustrates the complexity of drought resistance mechanisms in two wheat (Triticum aestivum L.) cultivars under dehydration and rehydration. BMC Plant Biol. 2016:16(1):188.

48. Rao KVM, Sresty TVS. Antioxidative parameters in the seedlings of pigeonpea (Cajanus cajan L.) in response to Zn and Ni stresses. Plant Sci. 2000;157(1):113-28.

49. Chavez-Salazar A, Bello-Perez LA, Agama-Acevedo E, Castellanos-Galeano FJ, Alvarez-Barreto Cl, Pacheco-Vargas G. Isolation and partial characterization of starch from banana cultivars grown in Colombia. Int J Biol Macromol. 2017:98:240-6

50. Chen LL, Chen QZ, Kong LQ, Xia FS, Yan HF, Zhu YQ, Mao PS. Proteomic and physiological analysis of the response of oat seeds to heat stress under different moisture conditions. Front Plant Sci. 2016;7:896.

51. Ma HY, Song LR, Shu YJ, Wang S, Niu J, Wang ZK, Yu T, Gu WH, Ma H. Comparative proteomic analysis of seedling leaves of different salt tolerant soybean genotypes. J Proteome. 2012;75(5):1529-46.

52. Zhou T, Li CY, Zhao W, Wang XR, Wang FQ, Sha JH. MaxReport: an enhanced proteomic result reporting tool for MaxQuant. PLoS One. 2016; 11(3):e0152067.

53. Zheng X, Fan S, Wei H, Tao C, Ma Q, Ma Q, Zhang S, Li H, Pang C, Yu S. iTRAQ-based quantitative proteomic analysis reveals cold responsive proteins involved in leaf senescence in upland Cotton (Gossypium hirsutum L). Int J Mol Sci. 2017:18:1984

54. Altschul SF, Gish W, Miller W, Myers EW, Lipman DJ. Basic local alignment search tool. J Mol Biol. 1990;215:403-10.

55. Wang $Y$, Wang X, Wang C, Wang R, Peng F, Xiao X, Zeng J, Fan X, Kang H, Sha $L$. Proteomic profiling of the interactions of $c d / Z n$ in the roots of dwarf polish wheat (Triticum polonicum L.). front. Plant Sci. 2016;7:1378.

56. Binns D, Dimmer E, Huntley R, Barrell D, O'Donovan C, Apweiler R. QuickGO: a web-based tool for gene ontology searching. Bioinformatics. 2009:25(22): 3045-6.

57. Kanehisa M, Sato Y, Kawashima M, Furumichi M, Tanabe M. KEGG as a reference resource for gene and protein annotation. Nucleic Acids Res. 2016:44(1):457-62

58. Liu SS, Liu YM, Jia YH, Wei JP, Wang S, Liu XL, Zhou YL, Zhu YJ, Gu WH, Ma H. GmI-MMP is involved in growth and development of leaf and seed, and enhances tolerance to high temperature and humidity stress in transgenic Arabidopsis. Plant Sci. 2017;259:48-61.

\section{Publisher's Note}

Springer Nature remains neutral with regard to jurisdictional claims in published maps and institutional affiliations.

\section{Ready to submit your research? Choose BMC and benefit from:}

- fast, convenient online submission

- thorough peer review by experienced researchers in your field

- rapid publication on acceptance

- support for research data, including large and complex data types

- gold Open Access which fosters wider collaboration and increased citations

- maximum visibility for your research: over $100 \mathrm{M}$ website views per year

At BMC, research is always in progress.

Learn more biomedcentral.com/submissions 